\title{
Extracting hidden rules from Brucellosis patients data in Razavi Khorasan province using association rule mining technique
}

\author{
Soheil Hashtarkhani1 \\ Medical Informatics Department, Mashhad University of Medical Science, Mashhad, \\ Iran \\ hashtarkhanis951@mums.ac.ir
}

\begin{abstract}
Brucellosis is a transmissible disease between humans and animals through infected animals and their products. The disease exists in most parts of the world especially in developing countries. Because of the serious impact of the disease in public health and socio-economical status, controlling the disease is very important in developing countries. The purpose of this article is to identify hidden patterns and relations between brucellosis patients which can be beneficial for physicians in diagnosis process. This study is a retrospective study of data collected from brucellosis patients of Razavi Khorasan province recorded at the health center, have been used. Due to differences in format and number of features collected during different years, before processing operations carried out in several stages to the same data. Fields associated with different methods and with expert opinion was converted into discrete fields and fields lost was estimated using the EM algorithm. APPIORI algorithm analysis was performed using the hidden relationships between data found that significant relationships were infected with expert opinion. Among the 163 relationship with over 7.0 Confidence rate which Weka software was discovered, by the application in consultation with an infectious disease expert, 10 clinically significant relationships were reported. Diagnosing brucellosis is really difficult to physicians because of its vague nature and symptoms. Because many unknown relationships between risk factors and demographic characteristics of the patients, the use of data mining concepts, especially in the medical data is beneficial because usually high volume assumptions are available. further studies can test the validity of these rules like Randomize Control Trial studies.

Keywords: Brucellosis, Data mining, Association Rule mining, Appriori.
\end{abstract}

\section{Declaration of conflicts}

This abstract is selected from the First International Congress of Diseases and Health Outcomes Registry and First National Congress of Medical Informatics, 14-17 February 2017, Mashhad, Iran

\section{Authors' biography}

No biography.

\section{References}

No references. 